\title{
Belt \& road organ donation capacity improvement cooperation training project (BROAOD)
}

\author{
Chloe Balleste ${ }^{1}$, Martí Manyalich ${ }^{1}$, Entela Kondi ${ }^{1}$, Hongtao Zhao ${ }^{2}$, Miao Pu ${ }^{2}$, Jie Zhao ${ }^{2}$, Yang Zhao ${ }^{2}$, You Wu ${ }^{2}$ \\ ${ }^{1}$ Department of International Cooperation, Donation and Transplantation Institute (DTI Foundation), Barcelona, Spain \\ ${ }^{2}$ Department of Education, China Organ Transportation Development Foundation, China
}

Background: China shows a continuous improvement in the practice of organ donation (OD) and transplantation (OT); reaching $4.16 \mathrm{pmp}$ in 2019. China Organ Transplantation Development Foundation (COTDF) in cooperation with the Donation and Transplantation Institute (DTI) Foundation have designed the BROAOD training program as a joint effort to grant intensive care doctors and other key donation professionals from China and Belt \& Road (B\&R) countries to international professional trainings.

Methods: Three training courses are scheduled to be performed online during the year. Interactive and dynamic sessions, including round tables, clinical cases, gaming, and small workgroups are design as course modality. The latest updates in the OD and OT field adapted to the needs of each of the participants countries will be used as educational materials. International models, detection, brain death, organ maintenance, family approach and organ evaluation are the main topics arrised. A questionnaire to evaluate the learning level is designed and distributed at the beginning and at the end of each course.

Results: Up to date, one edition of the course has been performed (May 2021); 55 participants have already been issued the BROAOD diploma. The expected total number of the trained professionals at the end of the course program will be more than 150 participants $75 \%$ from China and $25 \%$ form the B\&R countries.

Conclusions: Promoting communication and cooperation between participants coming from different countries, exchange of the clinical experience and enhance the abilities of detecting and referring potential OD are the key elements to improve the OD and OT activities. This international cooperation initiative promoted by the COTDF and DTI Foundation is an excellent example of how to support the global community to reach self-sufficiency in OD and OT. 